\title{
Leitartikel
}

\section{Pathologie der Darmmotorik}

\author{
Norbert Kopf
}

\author{
Meinem verehrten Lehrer Prof. Dr. Dr. h.c. Bernhard Huskamp \\ anlässlich des nach ihm benannten Koliksymposiums in Berlin, 7.-10.Juni 2012 gewidmet
}

\section{Vorbemerkungen zur Ätiologie}

Für die Ätiologie der meisten Magen- und Darmkoliken des Pferdes nehmen nach Meinung der "Wiener Schule" (Gratzl E. 1937 und 1942, Jaksch 1982) die Larven des 4. Stadiums von Strongylus vulgaris eine zentrale Stellung ein. Sie schmarotzen in der vorderen Gekrösearterie und der Bauchschlagader, schädigen dort nicht nur die Intima (Endarteriitis), sondern auch die ganze Arterienwand (Aneurysma). Diese Hypothese erhielt, quasi als Zusatzergebnis, durch Untersuchungen über die Wirksamkeit von Ivermectin (Slocombe et al. 1982) neve Nahrung: In angiographischen Studien an Fohlen, die experimentell mit Larven von Strongylus vulgaris infiziert worden waren, konnte röntgenologisch dargestellt werden, welche umfangreichen Veränderungen die Gekrösegefäße bereits kurze Zeit nach massiver Invasion durch diese Wurmlarven erleiden können. Die entzündlichen Veränderungen können nach Olt (1932) durch Zubildung von Bindegewebe auch auf die $\operatorname{der} A$. mesenterica cranialis angelagerten Bauchganglien übergreifen (Perineuritis). Auch Möllmann (1932) fand Entzündungsprozesse und Degeneration der Ganglienzellen bei über 83\% jener Pferde, die ein deutliches Gefäßaneurysma aufwiesen, während bei Pferden mit geringgradigen Veränderungen im Bereich der Gekrösegefäße diese krankhaften Prozesse der Bauchganglien in weniger als $30 \%$ der untersuchten Fälle nachgewiesen werden konnten. Die Schädigung der sympathischen Ganglien wird von Gratzl als Grundelement für die Neigung mancher Pferde zu vegetativer Imballance im Sinne einer Parasympathikotonie und der daraus resultierenden Motilitätsstörungen des Darmes (klonische Krämpfe, Dysperistaltik, Spasmus) angesehen. Hingegen bleiben selbst hochgradige Gefäßaneurysmen bezüglich der Blutversorgung des Darmes zumeist symptomlos. Von der Endarteriitis ausgehende Thrombosen verursachen nur relativ selten Embolien der Darmarterien in solchem Umfang, dass es zu akuter Mangeldurchblutung größerer Darmabschnitte, der sogenannten embolisch-thrombotischen Kolik, kommt. In der "Pionierzeit" der Kolikforschung wurden von Marek (1907) in systematischen Experimenten an Versuchspferden durch Unterbindung einzelner und mehrerer Gekrösearterien in verschiedenen Kombinationen jene Voraussetzungen erforscht, die eintreten müssen, damit ein Darminfarkt zustande kommt. Durch diese Arbeit wurden das pathoanatomische Erscheinungsbild und der zeitliche Ablauf bei gänzlichem oder teilweisem Verschluss der arteriellen Blutzufuhr an verschiedenen Darmabschnitten mit dem klinischen Verlauf von Embolien der Gekrösearterien vergleichbar. Erscheinungen der akuten Mangeldurchblutung von Darmteilen entstehen nur dann, wenn gleichzeitig mehrere benachbarte, miteinander anastomosierende Arterien ver- schlossen werden. Nach Pilwat (1910) und Joest (1971) entsteht ein anämischer Infarkt auch, wenn multiple Embolien der Arterien in einem größeren Areal vorliegen oder wenn eine etagenförmige Embolie eines Hauptgefäßes, z.B. Grimmdarmarterie, vorliegt. Der anämische Infarkt ist durch eine scharf begrenzte ischämische Nekrose gekennzeichnet, die von einer hämorrhagischen Randzone umgeben ist. Wird die Blutzufuhr nur größtenteils oder langsam unterbrochen, so entsteht das Bild der hämorrhagischen Infarzierung, da durch die Hypoxie die Gefäßwände geschädigt und durchlässig werden, wodurch es zum Blutaustritt in das Gewebe kommt. Bei noch geringerer, jedoch erheblicher Behinderung der Blutzufuhr tritt Ödemisierung der Darmwand -am stärksten im Bereich der Submukosa- ein. Sind nur kleinere Bezirke von der Mangeldurchblutung betroffen so erweitern sich kleine anastomosierende Gefäße rasch genug und in ausreichendem Umfang. In solchen Fällen ist Spontanheilung des hochgradig schmerzhaften hypoxämischen Krampfes des betroffenen Darmabschnittes naturgemäß möglich.

Umfangreiche Sektionsstatistiken zu Beginn des 20.Jahrhunderts (Wall 1908) und (Pilwat 1910) zeigen, dass die embolisch-thrombotische Kolik als Todesursache schon zu jener Zeit relativ selten beobachtet wurde, als die modernen Antiparasitika noch nicht zur Verfügung standen. So war ihr Anteil bei 834 Koliksektionen Walls nur 1,2\% und bei 423 Zerlegungen Pilwats 4,9\%. Diese relative Seltenheit des Darminfarktes stand nur scheinbar im Widerspruch zur Häufigkeit des Aneurysmas, da ja jeder Darmteil eine mehrfach gesicherte Blutzufuhr aufweist. Im Bereich der Blind- und Grimmdarmarterien wiesen Dobberstein und Hartmann (1932) ein Netz von anastomosierenden Seitenästen nach, das die entsprechenden Venen umspinnt. Dadurch wird verständlich, dass selbst ein kompletter Verschluss eines dieser Hauptgefäße durch einen kurzen Embolus nur geringe und vorübergehende Auswirkung auf die Blutversorgung haben kann.

Hartmann (zit. nach Gratz) hat bei 50 Pferdesektionen tödlich verlaufender Koliken, bei denen gleichzeitig ein Aneurysma vorlag, nur 4 embolisch thrombotisch verursachte festgestellt. Der Rest dieser Sektionen wies Verstopfungen des Zäkums und des Kolons nach. Gratzl sieht in dieser Aufteilung einen indirekten Beweis für die eingangs erwähnten Ergebnisse Möllmanns, dass die weitaus größere Bedeutung von Strongylus vulgaris im Rahmen des Koliksyndroms der Störung der autonom gesteverten Darmtätigkeit infolge von Entzündungsprozessen in der Gekrösewurzel zukommt: Die Neigung bestimmter Pferde (sogenannter Koliker), auf äußere Reize mit übersteigerter, schmerzhafter Peristaltik zu reagieren, wird als Über- 
empfindlichkeit eines labilen Vegetativums erklärt. Auch eine ständige Überreizung durch chronische Schädigung der Innervation wird als Ursache der ständigen leichtgradigen Hypertonie der Muskulatur der Darmwand mit folglicher Hypertrophie - wie für chronische Kolikformen wie z.B. die Blinddarmverstopfung kennzeichnend - angesehen. Aber auch akute, kurzfristige Mangeldurchblutung infolge von Gefäßkrämpfen oder von subtotalen Embolien kann durch $\mathrm{CO}_{2}$-Anhäufung im Blut zu krankhaft übersteigerter Peristaltik führen.

Die parasitär bedingte Schädigung der Innervation sowie der Blutversorgung des Darmes wird bis heute im Rahmen des Koliksyndroms als der Ausgangspunkt für die verschiedenen Verlaufsformen dieses Krankheitskomplexes angesehen (Huskamp et al. 1983, Jaksch 1982). Enteritiden parasitärer sowie mikrobieller Genese stellen eine weitere Ursache für die Entstehung peristaltischer Dysfunktion dar, welche als hauptsächliche pathogenetische Grundlage für die Entstehung von Darmverlagerungen anzusehen ist. Diese Erkenntnis wirkte sich auch auf das Therapiekonzept bei der Behandlung von Kolikpferden aus: Die Konsequenzen der oben dargestellten Sichtweise führten dazu, dass Drastika als kontraindiziert erkannt wurden und nicht mehr eingesetzt werden durften. Sie mussten der Motilitäts-regulierenden Therapie durch Spasmolytika und Analgetika (z.B.: Novalgin ${ }^{\circledR}$, Buscopan comp ${ }^{\circledR}$ ) Platz machen. (Peristaltika, wie Physostigmin, werden nur mehr bei atonischen Zuständen - z.B. post op. Paralytischer lleus - eingesetzt.)

Gratzl (1952) stellte fest, dass... „seitdem Drastika in der Koliktherapie nicht mehr verwendet wurden, an der I. Medizinischen Klinik in Wien der Anteil tödlicher Komplikationen (Rupturen und Darmverlagerungen) von 5,3\% (1926-1935) auf 2,1\% (1940-1951) gesunken war." Er sah dies als weiteres Indiz dafür an, dass die überschießenden Darmbewegungen in der ersten Phase des sog. katarrhalischen Darmkrampfes die entscheidende Rolle für die Entstehung der verschiedenen Ileussituationen des Pferdes spielen.

\section{Anatomische Besonderheiten des equinen Magen- Darmtraktes}

Erst bei fehlerhafter Bewegung des Darmes und bei unphysiologischer Verteilung des Darminhaltes - bedingt durch Beschleunigung und/oder durch Stauung - wirkt sich die eigentümliche Anatomie des Magen-Darm-Traktes des Pferdes für die Entwicklung von Darmverlagerungen begünstigend aus: Die schiefe Einmündung der Speiseröhre in den relativ kleinen Magen, wodurch Druckentlastung durch Erbrechen vereitelt wird, die große Beweglichkeit des langen Dünndarms, die sein nach aboral immer länger werdendes Gekröse zulässt. Die geringe Fixierung des voluminösen Colon ascendens und Caecums welche kleinflächig - fast punktförmig- mit der dorsalen Bauchwand verlötet sind und nur durch das große Oval der Körperhöhle in ihre normale Lage geordnet werden. Es liegt daher auf der Hand, dass die physiologische Lagerung des Darmes im Abdomen der unentwegten aktiven Erhaltung eines Gleichgewichtszustandes durch harmonische Darmtätigkeit bedarf. Durch ständige Wechselbeziehung zwischen Füllung und Peristaltik der einzelnen Darmabschnitte, aber auch durch die Wechselwirkung benachbarter Darmteile zur Körperwand und aufeinander wird dieses Gleichgewicht erreicht.
Auch der plötzliche Übergang von weiten in sehr enge Darmabschnitte wird schon immer als Disposition der Equiden für Störungen der Darmpassage diskutiert, doch erst bei Verkrampfungen, organischen Veränderungen (Verwachsungen und Narben) in diesen Bereichen oder durch unphysiologische Beschaffenheit des Darminhaltes kommt es zur Kotstauung (Obstipation) oder zum Verschluss (Obturation).

Weiters sind Form, Größe, Funktion und Aufhängung der Milz als anatomische Eigenheit des Pferdes zu nennen, die unter bestimmten Voraussetzungen als Angelpunkt für das Zustandekommen und die pathologische Aufrechterhaltung einer Grimmdarmverlagerung und -einschnürung anzusehen sind (Huskamp und Kopf 1980, 1983).

Pathoanatomische Dispositionen für Darmeinklemmungen Individuelle Dispositionen für Darmverlagerungen stellen die abnorme Weite anatomisch bedingter Lücken in der Körperwand (Ostium abdominale des Proc.Vaginalis) oder im Gekröse (Foramen omentale) oder infolge von Hemmungsmissbildungen vorhandene Pforten (Nabelbruch, nicht vollständige zurückgebildete Dottersackarterien bzw. ihr Gekröse dar (Kopf et al. 1983).

Erworbene Gekröseläsionen infolge von überstandenen Koliken kommen auch in Betracht (Kopf et al. 1979): Bei Hyperperistaltik ist es vorstellbar, dass bei temporär auftretenden Invaginationen, die sich wieder lösen, das Gekröse des Intussusceptums an der Kante des Intussuscipiens gezerrt wird und einreißt. (Huskamp ,1976, 1977, hat temporäre Dünndarminvaginationen während Pferdelaparotomien als Zufallsbefund festgestellt. Bei größeren Mastdarmvorfällen von $30 \mathrm{~cm}$ und mehr entstehen Einrisse des Mastdarmgekröses durch einen ähnlichen Mechanismus).

Traumen durch Sturz, Schwergeburt, Deckakt, sowie Narbenspangen nach Bauchhöhlenoperationen kommen ebenfalls in Betracht (Kopf et al. 1979).

\section{Physiologischer Gleichgewichtszustand der Eingeweide}

Tauchgleichgewicht

Wie Beobachtungen an normalen Schlachtpferden zeigen, nehmen die wegen ihrer langen Gekröse gut beweglichen Darmteile (Jejunum, Colon ascendens und Colon descendens) normalerweise eine stark variierende Lagerung ein. Teile der genannten Darmabschnitte können praktisch jeden Ort der Bauch- und Beckenhöhle erreichen. Jeder Darmteil kann sich widerstandslos und frei bewegen und einen seiner momentanen Füllung und Funktion entsprechenden Platz einnehmen. Neben der Eigentätigkeit der Darmwand sind dafür auch die Tätigkeit benachbarter Darmteile, das der Bewegung Grenzen setzende Gekröse und die den eiförmigen Raum der Bauchhöhle begrenzende Körperwand für Grad und Richtung dieser Bewegungen maßgebend. In dem allseits geschlossenen System der Bauchhöhle herrschen die Gesetze des Tauchgleichgewichtes nach dem Archimedischen Prinzip: Da die Darmwand und der vorwiegend wässrige, dünnbreiige bis weichteigige Darminhalt annähernd das gleiche spezifische Gewicht haben, können sich die normalerweise nicht prall, sondern kaum (Dünndarm) oder nur 
locker (Colon ascendens und Caecum) gefüllten Eingeweideteile zwischen den sie umgebenden schwebend mit äußerst geringem Kraftaufwand bewegen. Größere Gasblasen befinden sich normalerweise nur im Saccus caecus ventriculi und in der Basis caeci, also an Orten, die ohnehin beim aufrecht stehenden Pferd an höchster Stelle fixiert sind. Festteigiger Inhalt befindet sich in der Regel nach der Aufnahme des Rauffutters im Fundus des durch seine kurzen Bänder weitgehend lagekonstanten Magens und in Form von Kotballen in kleine Portionen aufgeteilt im kleinen Kolon, wodurch die Beweglichkeit des Enddarms nicht eingeschränkt ist. Eine größere Masse festen Kots befindet sich vor der Defäkation lediglich in der lagekonstanten Ampulla recti.

Es kann also jeder bewegliche Darmabschnitt mit nur geringem Energieaufwand im "freien" Raum der Bauchhöhle in alle Richtungen bewegt werden, ohne an ein Hindernis zu gelangen. (Anlässlich der Rektaluntersuchung gesunder Pferde kann man sich davon überzeugen, dass der gestreckte Arm allseits bewegt werden kann, ohne dass ein unbewegliches Hindernis diese Bewegung einschränkte. Da zwischen den Eingeweiden nur ein kapillarer Spalt besteht, der von äußerst spärlicher Bauchhöhlenflüssigkeit erfüllt ist, liegł auf der Hand, dass der Platz, den ein Darmteil verlässt, im selben Augenblick von einem benachbarten eingenommen werden muss. Die physiologische Motilität des Darmes ist also eine sehr ökonomische Kombination von aktiver Eigenbewegung des Darmes und passivem Bewegt-Werden durch sich bewegende, benachbarte Darmteile und von füllungsbedingtem Absinken und Emporsteigen.

\section{Adhäsionskräfte}

In Anbetracht der beachtlichen Masse des frei beweglichen Teiles des gefüllten Darmkonvolutes könnte die Vorstellung aufkommen, dass bei raschen Drehungen und Wendungen des Tieres die Bewegungen der Eingeweide hinter denen des Körpers zurückbleiben und dadurch Verdrehungen entstehen könnten. Tatsächlich wird in vielen Abhandlungen dem Wälzen der Tiere für die Entstehung der Darmverlagerungen große Bedeutung beigemessen. Unter normalen Verhältnissen machen die Eingeweide, trotz hoher Masse und großer Verschieblichkeit, alle Bewegungen des Tieres, auch die raschesten Wendungen mit. Dafür sorgen die Adhäsionskräfte zwischen den Exkavationen der Körperwand und den kongruent anliegenden Darmteilen, die nur gemäßigtes Gleiten entlang der Körperwand, nicht aber plötzliche Bewegungen in anderer Richtung erlauben. Diese Kräfte sind auch zwischen den Eingeweiden wirksam.

So kann man sich bei der operativen Lageberichtigung voluminös gefüllter Dickdarmteile davon überzeugen, wie groß diese "Sogkräfte" sind: Die gefüllten Kolonlagen sind aus der Höhlung, in der sie liegen, nicht heraus zu bewegen, wobei es gleichgültig ist, ob ihr Inhalt feste Konsistenz aufweist oder flüssig ist. Erst wenn man größere Flüssigkeitsmengen in die Bauchhöhle einfüllt, wodurch die kapilläre Adhäsion zur Körperwand aufgehoben wird, gelingt die Vorlagerung.

Die Adhäsionskräfte sowie die Masse der - zwangläufig jeder Bewegung eines Darmabschnittes folgenden - benachbarten
Darmteile dämpfen bzw. verhindern somit rasche Ortsveränderungen der Eingeweide.

\section{Pathophysiologie der Darmverlagerung}

\section{Störungen des Gleichgewichtszustandes}

Die folgende Darstellung basiert auf einer Analyse der Krankengeschichten, der Operations- und Sektionsberichte von Kolikpferden, sowie auf ergänzenden Experimenten an frisch geschlachteten Pferden, bei denen verschiedene Vorgänge simuliert werden konnten.

\section{Abnahme der Adhäsionskräfte}

Die Adhäsionskräfte nehmen -wie oben beschriebendadurch ab, wenn sich mehr Flüssigkeit zwischen den verschiedenen Darmteilen bzw. zwischen den Eingeweiden und der Bauchwand ansammelt (z.B. vermehrte Peritonealflüssigkeit während der Gravidität, Hydrops ascites, Transsudat).

Ein Effekt, den man sich, wie gesagt, bei der operativen Lageberichtigung zunutze machen kann, indem man reichlich isotone Kochsalzlösung in die Bauchhöhle gießen lässt (z.B. Reposition des verlagerten großen Kolons).

\section{Auftriebskräfte}

Bei gleichzeitigem Auftreten von Koteindickung (z.B. Obstipationen) und größeren Gasansammlungen (Meteorismus) geht das Tauchgleichgewicht verloren: Nach dem Archimedischen Prinzip haben gashaltige Darmteile jenen Auftrieb, der dem Gewicht jener flüssigkeitsgefüllten Darmabschnitte gleichkommt, welche sie verdrängen (d.h. etwa $1 \mathrm{~kg}$ Auftrieb pro 1 I Darmgas). Es wirken also in diesem Zustand enorme Kräfte auf geblähte Darmteile ein, welche rasche, passive Bewegungen erzwingen, wenn sich die Tiere wälzen oder rasch bewegen. Diesen Kräften können sie um so leichter folgen, je mehr Flüssigkeit sich im Bauchraum befindet. Das Wälzen während eines Kolikanfalles kann also die Entstehung von Darmverlagerungen begünstigen.

Die Auftriebskräfte können aber auch bei der sog. Wälzmethode zur Lageberichtigung des in den Milznierenraum verlagerten und dort eingeschnürten Colon ascendens therapeutisch genützł werden (Kopf 1997).

\section{Expansions- und Strangulationskräfte}

Andererseits führt die durch Meteorismus wie auch die durch Überfüllung mit festem Inhalt bedingte Expansion eines Darmabschnittes zu dessen Verkeilung im begrenzten Raum der Bauchhöhle. Je nach Schicksal des Falles kann dies sowohl der Entwicklung von Darmverlagerungen entgegen wirken (z.B.: Obtipation der linken ventralen Längslagen des großen Kolon). Andererseits kann durch Blähung die spontane Reposition bereits entstandener Lageveränderungen verhindert werden (z.B.: Dislocatio coli asc.) Die Expansionskräfte üben einen wachsenden Druck auf die Körperwand, von außen an der Vorwölbung der Flanken erkennbar, sowie auf die - evtl. 
ebenfalls geblähten - benachbarten Darmteile aus. Die Spannung auf der Darmwand und dem gefäßführenden Gekröse konzentriert sich an Knick- und Strangulationsstellen punkförmig, d.h. der Flächedruck ist dort besonders groß. An Drehstellen kommen durch die Wringbewegung nach den Hebelgesetzen Kräfte zur Wirkung, die die Dehnungskräfte der Darmwand des geblähten und gedrehten Darmteiles um ein Mehrfaches übersteigen, sodass -selbst bei hochgradigem Meteorismus- kein Gas mehr entweichen kann.

\section{Fangkorb- und Reusenmechanismen}

Durch Blähung können auch normalerweise kollabierte Bauchfellnischen und Aussackungen aufgeweitet werden und nehmen dann eine die Inkarzeration ermöglichende trichterartige Form ein.

\section{Spatium lienorenale}

Der Spalt zwischen Milzmagen- bzw. Milznierenband (kaudal), dem Magen und der Niere (medial) und der Milz und dem Zwerchfell (lateral) nimmt bei Erweiterung die Form eines schlanken Trichters an, dessen kleinere Öffnung durch das scharftkantige Milznierenband und die dorsale Bauchwand begrenzt nach dorsokaudal gerichtet ist.

\section{Vestibulum bursae omentalis - Foramen omentale}

Der Raum zwischen Magen (kranial), magenähnlicher Erweiterung des Colon ascendens (kaudoventral) und der dorsalen Bauchwand (Leber) ist bei Aufweitung ebenfalls trichterförmig, wobei die kleinere Öffnung, nach dextrodorsal gerichtet, vom scharfkantigen Foramen omentale gebildet wird. Diese Formation konnte sogar laparoskopisch in vivo dargestellt werden (Fischer 1997).

Die beiden oben beschriebenen Räume wirken durch ihre konische Form wie reusenförmige Fangkörbe, wenn eine Darmschlinge durch die weite Öffnung eintritt: Der Durchtritt durch die enge Öffnung am "Ausgang des Trichters" gelingt relativ leicht, doch ein Zurückziehen ist, zumindest in gefülltem Zustand, unmöglich. Aus diesem Grunde ist der Eintritt von Darmschlingen über die engere Öffnung dieser trichterförmigen Räume sehr viel unwahrscheinlicher. Tatsächlich fanden Huskamp und Kopf (1980) unter 122 Verlagerungen des Colon ascendens in den sogenannten Milznierenraum nur eine einzige, bei der sich der Darm von hinten nach vorne über das Milznierenband schob. Weiters stellten sie fest, dass die durch Stauung vergrößerte Milz bei dieser Darmverlegung wie ein Anker das seitliche Herausgleiten der verlagerten Kolonschleife aus dem Milznierenraum verhindert, so dass das Zustandekommen der abnormen Fixierung des Grimmdarmes dem Einhaken eines Taves in einen Karabinerhaken vergleichbar erscheint.

Auch bei der häufigen Einklemmung des Dünndarmes im Foramen omentale s. epiploicum ist die sogenannte laterale Form, in welcher der Darm von rechts durch das Foramen omentale tritt, eine Rarität (Huskamp et al.1980). Der Dünndarm tritt in der Regel, das Omentum majus vor sich her- schiebend, von medial in das relativ weite Vestibulum omentale ein -indem er es aufweitet- und wird dann durch das scharfkantig begrenzte Foramen omentale nach rechts-lateral hindruchgepresst. Sobald sich die durchgetretene Darmschlinge mit Flüssigkeit füllt, was durch die venöse Stauung beschleunigt wird, ist ein Zurückgleiten i.d.R. nicht mehr möglich.

Anulus vaginalis, sanduhrförmige Einziehung des Processus vaginalis

Einen ähnlichen Inkarzerationsmechanismus birgt der Bau des Processus vaginalis in sich: Der Bauchring (Anulus vaginalis) - normalerweise eine durch eine mediale Bauchfellfalte verdeckte Spalte - kann relativ weit sein (3 bis $6 \mathrm{~cm}$ ), der Scheidenhauthals verjüngt sich die ersten 3 bis $4 \mathrm{~cm}$ seines Verlaufes trichterförmig bis zur sogenannten sanduhrförmigen Einziehung und erweitert sich danach zum Cavum vaginale (Überreiter 1958). Bei Blähung desselben entstehen - wie wir im Experiment an Schlachtpferden feststellen konnten - im Bereiche dieser Engstelle an der kranialen Kontur zirkuläre Falten (ähnlich einem hochgeschobenen Hemdsärmel) und engen ihre Lichtung zusätzlich ein (Zechner 1986, Kopf und Zechner 1988). Dem technischen Fortschritt entsprechend konnten diese Vorgänge von Huskamp laparoskopisch beobachtet und in vivo gefilmt und dargestellt werden. Die Kenntnis dieser Vorgänge konnte sogar für die unblutige Reposition einer inkarzerierten Dünndarmschlinge genütz† und laparoskopisch beobachtet werden: Die Reposition gelang, indem der - durch die eingetretene Darmschlinge aufgeweitete- Scheidenhauthals durch hohen manuellen Druck von außen in die Bauchhöhle vorgestülpt - also gleichsam wie ein Hemdsärmel bis zur sanduhrförmigen Einziehung umgekrempelt- wurde. Dadurch wurde der Trichtereffekt umgekehrt, und die Darmschlinge konnte in die Bauchhöhle zurückgepresst werden (Huskamp und Kopf 1997). Dieser Vorgang ist freilich nur in sehr frischen Fällen möglich, und wird wohl kaum routinemäßig eingesetz† werden können. In der Regel ist die Herniotomie der Engstelle erforderlich, um die Reposition zu ermöglichen. Die Spannung am Inkarzerationsort kann in Einzelfällen so groß werden, dass quer verlaufende Rupturen des Scheidenhauthalses mit anschließender Eventration des inkarzerierten Darms unter die Skrotalhaut entstehen können.

\section{Pathologische Darmbewegungen}

Aktive Darmbewegungen

Neben der eingangs erwähnten Hyperperistaltik, die sich zum klonischen Darmkrampf steigern kann, kommen noch Koordinationsstörungen im Ablauf der peristaltischen Wellen in Betracht. Es besteht die klinische Beobachtung überlauter, pfeifender Darmgeräusche, die auf die beschleunigte Passage spastisch verengter Darmteile hinweisen und die abrupt enden, was darauf deutet, dass die Passage durch den steigenden Tonus schließlich gänzlich unterdrückt wird. Dieser Zustand lässt sich im Experiment am Versuchspferd leicht durch die Injektion von Azetylcholin in eine Dünndarmarterie demonstrieren. Ein spastisch verengter Dünndarmschenkel kann sich auf Fingerstärke kontrahieren, bekommt dann derb-elastische Kon- 
sistenz und streckt sich geradlinig aus (Darmsteifung). In frischen Fällen von katarrhalischem Darmkrampf kann man solche spastisch kontrahierte Dünndarmteile rektal tasten. Je nach Grad und Daver eines solchen Spasmus wirkt der kontrahierte Darmabschnitt als Hindernis, als düsenartige Verengung oder als Angelpunkt für Verknotungen.

Bei anhaltender Heftigkeit der Darmtätigkeit des davor liegenden prästenotischen Darmabschnittes können diese verschiedenen Wirkungen auch verschiedene Lageveränderungen zur Folge haben.

Treibende Kräfte drängen das Hindernis, das zusätzlich auch aus einer Obstipation von der Verkrampfung bestehen kann, immerfort in eine Richtung, so dass der Dünndarm im Oval der Bauchhöhle um seine Gekröseachse regelrecht aufgespult werden kann: Es entsteht ein Volvulus mesenterialis, dessen Form unzweifelhaft zu erkennen gibt, dass die Drehung von einer Darmschlinge ausging. Nach Erlahmen der Hyperperistaltik sorgt der sich entwickelnde prästenotische Meteorismus dafür, dass immer mehr Darmschlingen in die Aufrollung hineinwandern.

Der Nachweis, dass die Dünndarmdrehung tatsächlich so entstehen kann, konnte dadurch erbracht werden, dass bei zwei Fohlen mit Volvulus mesenterialis im poststenotischen Darmschenkel ein bleibendes Hindernis aufgefunden werden konnte: In einem Falle eine spastische Obstipation, die nicht durch die Darmverlagerung zu erklären ist, im anderen Falle eine Invagination.

Es wurden aber auch eine Reihe von Fällen beobachtet, bei denen der poststenotische Dünndarmschenkel leer war, so dass auch noch die Möglichkeit in Betracht zu ziehen ist, dass düsenähnliche Verengungen infolge der beschleunigten Fortbewegung des Darminhaltes Rückstoßkräfte erzeugen, wodurch der betreffende Darmteil über längere Zeit im Kreis getrieben wird und die davor liegenden - sich in zunehmendem Maße blähenden - Darmschlingen zur Aufrollung kommen.

Im Experiment kann dieser Vorgang ganz einfach simuliert werden: Der exenterierte Dünndarm wird an einem Ende durch die Ligatur eingeengt und das Darmrohr mit Wasser durchflutet. Die Schlingerbewegungen des eingeengten Endes werden heftiger, wenn das Wasser ruckweise auf- und abgedreht wird, und die Rückstoßkräfte werden in eine Kreisbewegung umgesetzt, wenn der Darm in ein ovales Plastikschaff gelegt wird.

Darmeinschiebung (Invagination) und Darmverknotung (Volvulus nodosus) sind die augenfälligsten Resultate von peristaltischer Dysfunktion: Bei der Invagination wird der spastisch versteifte Darmschenkel in den sich erweiternden folgenden Darmabschnitt hineingetrieben. Dieser reagiert - vermutlich infolge allgemein übersteigerter Erregbarkeit auf Dehnungsreize - auf diesen festen Inhalt wie auf einen Fremdkörper und versucht das Intussuszeptum immer weiter nach aboral zu drängen, bis schließlich ein Daverspasmus an der Eintrittspforte des Intussuszeptums die bleibende Fehllagerung bewirkt. Durch die anschließende Ödemisierung des eingedrungenen Darmteiles wird die Irreversibilität dieser Darmverlagerung besiegelt.
Beim Volvulus nodosus müssen durch die hyperperistaltisch erzeugten Rückstoßkräfte Schlinger-, Taumel- und Trudelbewegungen entstehen, deren Ablauf im Bereich eines spastisch versteiften Darmabschnittes gestört wird, da die Flexibilität desselben nicht in vollem Umfang gegeben ist. Ähnlich einer Fahne, die sich im Sturm schließlich um den Mast schlingt, laufen die prästenotischen Darmschlingen so lange gegen dieses Hindernis an, bis es wie ein Knebel im Gekröse des oral gelegenen Darmabschnittes hängen bleibt, wenn sich dieser achterförmig darum schlingt. Der entstehende prästenotische Meteorismus zieht den Knoten fest und kann ihn noch eine Strecke in aboraler Richtung weiterschieben. Bei Berstungen des Gekröses werden in der Folge noch mehr Darmschlingen in die Verknotung einbezogen, so dass schließlich kaum geklärt werden kann, ob die Verknotung anlässlich des Gekröserisses entstand, oder ob die Gekröseläsion eine Folge der Verknotung darstellt, sofern die Ränder der Gekröseläsion nicht schon vernarbt sind (Hernia mesenteralis).

Auch ein anderer Darmteil kann zum Drehpunkt für Dünndarmschlingen werden: z.B. Volvulus mesenterialis jejuni et coli descendentis, eine Verschlingung beider, Darmteile mit langem Gekröse. Alle genannten Lageveränderungen des Darms, die auf Hyperaktivität und Dysfunktion der Darmbewegungen zurückzuführen sind, werdengehäuft beim Fohlen und Jährling beobachtet. Fast immer kann zusätzlich eine Enteritis z.B. im Zusammenhang mit Askaridenbefall nachgewiesen werden. Die auftretenden Kräfte sind nachweislich so groß, dass spontan Darm- und Gekröserupturen entstehen können (Kopf 1976)

\section{Passive Darmbewegungen}

Segmentierung des Darmkanals

Im Unterschied zum Obturationsileus (Darmverlegung oder Verstopfung), bei dem in der Regel nur ein prästenotischer Darmschenkel, in dem sich vor dem Hindernis der Darminhalt staut, und ein leerer poststenotischer Darmschenkel zu unterscheiden sind, liegen bei Strangulationsileus drei pathophysiologisch zu unterscheidende Darmabschnitte vor, da das Darmrohr durch die Lücke, den Strang, die Knickung oder Drehung oder die Invaginationspforte an zwei verschiedenen Stellen eingeengt wird. Den Darmabschnitt zwischen diesen beiden Einengungen bezeichnen wir als stenostenotischen oder interstenotischen Darmabschnitt.

Im prästenotischen Darmabschnitt kommt es zur Stauung des Darminhaltes und infolge von Zersetzungsvorgängen rasch zum Meteorismus. Da eine unmittelbare Behinderung der Blutversorgung fehlt, treten Darmwandschädigungen erst im fortgeschrittenen Stadium durch die Dilatation und die Wirkung von Toxinen auf.

Im stenostenotischen Darmabschnitt wird ebenfalls der Inhalt aufgestaut und es entsteht gleichzeitig und mit der Inhaltsstauung in zunehmendem Maße eine Behinderung des venösen Abflusses, sodass durch Blutstauung in diesem Darmteil zusätzlich die Flüssigkeitssequestration (Transsudation) in das Darmlumen beschleunigt wird. Daher ist der Darmabschnitt zwischen den Stenosen neben dem wesentlich rascheren Fortschreiten der Darmwandschädigung - im Vergleich zum prä- 
stenotischen - auch einer rascheren Expansion unterworfen, welche durch die vermehrte Entstehung von Darmgasen eine zusätzliche Beschleunigung erfährt.

Der poststenotische Darmschenkel ist in der Regel leer und ohne Zirkulationsstörung, außer er ist so stark in die Einklemmung oder Drehung hineingespannt, dass die entstehenden Dehnungskräfte eine mechanische Schädigung bewirken. Die durch den prästenotischen und stenostenotischen Meteorismus wirkenden Expansionskräfte führen zu bestimmten passiven Darmbewegungen, die folgende Gesetzmäßigkeiten aufweisen:

\section{Einsaugen}

Durch die Aufblähung zwischen den beiden Hindernissen neigt der stenostenotische Darmteil dazu, den leeren, poststenotischen Schenkel in die Einklemmung oder Drehung hineinzuziehen, bis dieser Vorgang am anatomischen Fixpunkt zum Stillstand kommen muss. Wall (1908) hat als erster auf dieses Phänomen hingewiesen. Beim Dünndarm ist dies das Ende des lleums am Caecum, beim Colon ascendens die Verlötung der magenähnlichen Erweiterung des Colon dorsale dextrum (Ampulla coli) mit der dorsalen Bauchwand bzw. beim Colon ventrale dextrum die Plica ceacocolica.

Tatsächlich liegt der aborale Schnürring bei über 90\% aller Dünndarmstrangulationen im Bereich des lleums, und zwar an jener Stelle, bis zu der sich Ileum und Caecum zum Ort der Einklemmung spannen lassen, Das sind beim Foramen omentale etwa $10 \mathrm{~cm}$, beim Ostium vaginale 20 bis $30 \mathrm{~cm}$. Die Spannung kann fallweise so groß werden, dass die Plica ileocaecalis einreißt. Bei Längsachsendrehungen des Colon ascendens überwiegen die fatalen Fälle von Torsio coli totalis, bei denen durch die Expansion die Drehstelle bis zur Anheftung der Kolonschleife hochgeschoben wurde., Im Vergleich dazu sind Fälle von Grimmdarmdrehungen, bei denen die Drehstelle im Bereiche der linken Längslagen oder der Querlagen liegt - also nur ein Teil der Längslagen gedreht ist (Torsio coli partialis) - weitaus seltener.

Ein weiteres Moment, wodurch - besonders in weiteren Lücken (z.B. Hernia omentalis) und Strangulationen durch Narbenstränge (Hernia pseudoligamentosa) - dieser Einsaugprozess gefördert wird, ist die Tatsache, dass die Einengung des Darmrohres zwischen stenostenotischem und poststenotischem Abschnitt immer größer ist als die oral davon gelegene Stenosierung zwischen prä- und stenosteontischem Darmteil. Das ist darauf zurückzuführen, dass die Abknickung des Darmrohrs durch einen Strang oder eine Kante zwischen den beiden geblähten Darmabschnitten aufgrund des hohen Druckes im Darmrohr nicht so stark erfolgen kann wie zwischen dem prallen stenostenotischen Darmteil und dem leeren poststenotischen, die durch die aborale Einschnürung getrennt sind. Diese Regel wird von Joest (1971) auch für das Schicksal von Darmeinklemmungen äußerer Hernien angegeben. Es ist somit möglich, dass noch eine zeitlang flüssiger und gasförmiger Darminhalt vom prästenotischen zum stenostenotischen Darmabschnitt übertreten kann und somit die Volumenzunahme desselben und damit auch die Vergrößerung der strangulierten Darmpartie beschleunigt wird.
Bei der Hernia diaphragmatica wird der passive Einsaugprozess aktiv durch den rhythmisch auftretenden Unterdruck im Thorax infolge der Atemtätigkeit zusätzlich verstärkt!

Diese Vorgänge vor Augen wird leicht erklärlich, warum orale Flüssigkeitsabgaben, vor allem jene von Glaubersalzlösung, das Fortschreiten einer lleuserkrankung - neben der Beschleunigung der Dehydration - auch auf mechanischem Wege beschleunigen! (Therapeutische Konsequenz ist die Kontraindikation salinischer Laxantien bei jeder Form von lleusverdacht!)

\section{Durchwandern einer Inkarzeration (Stafettenlauf)}

Bei der Hernia inguinalis incarcerata des Hengstes ist die Darmeinklemmung naturgemäß früher feststellbar als bei inneren Hernien. Die Tiere kommen daher im Durchschnitt wesentlich früher zur Operation als Kolikpferde mit innerer Dünndarmeinklemmung. Anläßlich der Herniotomie des Processus vaginalis kann in frischen Fällen (2. bis 6. Krankheitsstunde) Jejunum als Bruchinhalt festgestellt werden, während in später eingelieferten (8. bis 10. Krankheitsstunde) häufig lleum eingeklemmt ist, welches in Relation zur Krankheitsdaver oft nur geringe Darmwandschädigung aufweisen kann. Bei in einigen Fällen zusätzlich vorgenommenen medianen Laparatomien -um zusätzliche Dünndarmverlagerungen auszuschließen- konnten im prästenotischen Jejunum mehrere zirkuläre Schnürstellen gefunden werden, die in unregelmäßigen Abständen angeordnet waren. Die aboralen waren zum Teil noch anämisch, bei den oral gelegenen hatten sich schon ringförmig angeordnete subseröse Blutungen gebildet. Es bestanden also deutliche Indizien dafür, dass der prästenotische Darm in dem Maße aus der Einklemmung auswanderte, als der poststenotische in die Hernie hineingezogen wurde. Für die abnorme Fixierung des stenostenotischen Darmteiles im Brustsack war also weniger der Klemmdruck im Bereiche des Schnürringes auf die Darmrohre, als die Knebelwirkung des Bruchinhaltes verantwortlich. Dieses Durchwandern der Hernie geht aber offensichtlich nicht fließend, sondern ruckweise vor sich, wie die verschiedenen Schnürringe beweisen. Dies wiederum ist ein Zeichen dafür, dass nicht nur die kontinuierlich zunehmende prästenotische Überfüllung das Auswandern dieses Darmschenkels bewirkt, sondern dass auch periodischer Druckanstieg - etwa durch gegen das Hindernis ankämpfende Darmbewegungen, oder auch Druckschwankungen in der Bauchhöhle beim Niederwerfen während eines Kolikanfalles - beteiligt ist. Voraussetzung für das Durchwandern einer Hernie ist, dass die Bruchpforte relativ klein, das Volumen des Bruchinhaltes relativ konstant bleibt und der Inhalt des eingeklemmten Darmteiles aus Flüssigkeit besteht, d.h. leicht beweglich, aber nicht zusammendrückbar ist.

Am eröffneten Abdomen eines frisch geschlachteten Hengstes konnte das Durchwandern einer experimentell erzeugten Hernia inguinalis simuliert werden: Über durch Tabaksbeutelnähte abgedichtete Stichinzisionen werden in den Leerdarm zwei Infusionsschläuche eingeführt, die etwa $50 \mathrm{~cm}$ voneinander entfernt frei enden. Der Darmabschnitt, der das aborale Schlauchende beherbergt, wird mit einem durch das Gekröse geführten Zügelfaden in den Processus vaginalis gezogen, Der Zügelfaden wird dabei mit Hilfe einer Sonde mit Öhr 
durch den Processus vaginalis nach Perforation desselben nach außen geführt. Einer der Infusionsschläuche endet somit im so erzeugten prästenotischen, der andere im stenostenotischen Darmteil. Über sie wird Wasser in das Darmlumen infundiert, und zwar in den eingeklemmten Darm etwas rascher, so dass dieser die begrenzte Höhle des Scheidenhauthalses prall anfüllt. Mit zunehmender Füllung des prästenotischen Darmschenkels beginnt der Darm in der beschriebenen Weise zu wandern, wobei die durch den Zügelfaden markierte Stelle alsbald prästenotisch erscheint und leerer Darm von poststenotisch einwandert (Kopf 1985).

Dieser Mechanismus stellt über eine gewisse Zeit einen Schutz für den eingeklemmten Darm dar, da - ähnlich einem Stafettenlauf - immer ein anderer Abschnitt von der Mangeldurchblutung betroffen ist.

\section{Entgegengesetzte Schubkräfte}

Beim mit verfestigtem Kot erfüllten Colon ascendens, bei dem bekanntlich zwei Darmrohre mit entgegengesetzter Richtung der Peristaltik fest miteinader verbunden sind, wirkt sich eine Einengung beider Darmrohre von außen (Torsion, Flexion und Strangulation) so aus, dass gegen das Hindernis von beiden Seiten jeweils ein Kotpfropf geschoben wird. Diese Schubkräfte sind zumeist nicht ganz gleich, da auch hier die Beobachtung gemacht werden kann, dass in der Regel die Passage vom prästenotischen Teil des Colon ventrale zum stenostenotischen Teil, der die Beckenflexur und ein mehr oder weniger großes Stück der ventralen und dorsalen Kolonlagen umfasst, weniger behindert ist als jene vom stenostenotischen Teil des Colon dorsale zum poststenotischen Teil desselben.

Die Schubkräfte können sich über längere Zeit fast vollständig aufheben, doch besteht die Tendenz des Einsaugens. Infolge der festen Konsistenz des Inhalts gehen alle Bewegungen wesentlich langsamer vor sich als bei flüssigem Inhalt und Gasbildung, doch ist die Spannung am Strangulationsort zumeist beträchtlich. Je nach Anordnung der Tänien und der Blutgefäße im Strangulationsort kommt es zur Drucknekrose auf den Bandstreifen oder zur Unterdrückung des venösen Abflusses, was zu subserösen Blutungen um die gezerrten Gefäße führt und Stauung im gesamten abgeschnürten Bereich bewirkt.

Am besten sind diese Vorgänge bei der Verlagerung des Colon ascendens in den Milznierenraum zu erkennen (Huskamp und Kopf 1980), doch kann die entgegengesetzte prästenotische und stenostenotische Kotstauung regelmäßig bei jeder anderen Einschnürung der Kolonschleife beobachtet werden (z.B.: Strangulation durch zerrissenes Omentum majus). Bei Torsionen des mit festem, nicht gärendem Inhalt erfüllten Grimmdarmes besteht die Chance, dass der voluminöse Kotstau im prästenotischen Colon ventrale ein Auflaufen der Drehstelle bis zur magenähnlichen Erweiterung verhindert . Dieses Gleichgewicht zwischen den Kräften ist die Erklärung für das Zustandekommen der Torsio coli partialis aber auch für andere Verlagerungen der Grimmdarmscheife (Retroflexio coli, Dislocatio coli asc. ad dextram).

Die therapeutische Konsequenz dieser Beobachtungen ist, dass durch die Eingabe von laxierenden Mitteln (Glauber- salzlösung, Paraffinum liquidum) zuerst der prästenotische Obstipationspfropf im Colon ventrale erweicht wird, was dazu führt, dass unter Umständen vermehrt breiiger Inhalt in den stenostenotischen Darmschenkel gelangt, während der stenostenotische Kotpfropf durch Ankurbelung der Wasserresorption gleichzeitig eine Verhärtung erleidet. Außerdem wird durch die Koterweichung der Widerstand der prästenotischen Kotansammlung gegen ein Einsaugen gemindert. Da diese Mittel also die Chance erhöhen, dass die von der Einschnürung betroffene Darmpartie größer wird, ist ihr Einsatz auch bei milde verlaufenden Dickdarmverlagerungen kontraindiziert.

\section{Ventilmechanismen des Magen-Darm-Kanals}

Magendilatation

Die bekannte, für das Pferd eigentümliche schräge Einmündung des Ösophagus in den Magen, welche eine Druckentlastung durch Erbrechen nach vorne in der Regel unmöglich macht, führt bei jeder Störung des Abflusses aus dem Magen zur akuten, sekundären Magenüberladung (Magendilatation). Die Abflussbehinderung ist sehr häufig reflektorisch durch Pylorospasmus bedingt, kann mechanisch durch Rükkstau, vielleicht auch durch Antiperistaltik (Dünndarmileus) hervorgerufen werden.

\section{Blinddarmdilatiation}

Bei Dilatation hat der Blinddarm die Eigenschaft, sich einzurollen, so dass sein Ausgang, das Ostium caecocolicum, schlitzförmig verschlossen wird und der Übertritt von Darminhalt vom Blinddarmkopf in den Anfangsteil des Colon ventrale dextrum (Vestibulum coli) auch dadurch erschwert wird, dass dieses vom sogenannten überhängenden Teil der Basis caeci zusammengedrückt wird (Gratzl 1934, Kopf 1982). Dieser passive Verschlussmechanismus besteht selbstverständlich auch beim prästenotischen Meteorismus des Blinddarms, falls ein Passagehindernis im großen Kolon besteht.

Versuche am frisch geschlachteten Pferd haben jedoch ergeben, dass bei Aufhebung dieser Sperre durch Punktion des Blinddarms im Colon ventrale angesammeltes Gas retrograd durch das nunmehr geöffnete Ostium caecocolicum in den Blinddarm entweichen kann.

Somit kann auch dem prästenotischen Meteorismus im Colon ascendens durch Punktion des Blinddarms - die sicher und einfach durchzuführen ist - begegnet werden. Diese Maßnahme ist somit geeignet, die Spannung am Strangulationsort wesentlich zu vermindern, was einen Zeitgewinn bezüglich des Grades und somit der Folgen der Zirkulationsstörungen im stenostenotischen Darmabschnitt bedeutet. Zumindest im Anfangsstadium einer Dickdarmverlagerung erhöht dies die Chance einer Spontanreposition.

Verschluss des Dünndarmes durch Paralyse

Nach der Phase der Hyperperistaltik wird häufig eine Phase der Darmlähmung - als Ausdruck eines Erschöpfungszustan- 
des - beobachtet. In dieser Phase entstehen beim Dünndarm mit seinem langen Gekröse weitere Passagehindernisse dadurch, dass sich die geblähten Darmschlingen im Abdomen verkeilen und verdrehen, dass zwischen den einzelnen Schlingen Dreh- und Knickstellen entstehen, die nunmehr auch ein mechanisches Passagehindernis darstellen.

Im Experiment am toten Pferd lässt sich der an seinem Gekröse hängende Dünndarm, in den man einen Schlauch eingeführt hat, nicht mit Wasser durchfluten. Am exenterierten Darmkonvolut dringt das Wasser auch unter Druck nur 2 bis $3 \mathrm{~m}$ vor. Wird der Dünndarm bei diesem Versuch in der Bauchhöhle belassen, so dringt mit Tierkohle gefärbtes Wasser eine noch kürzere Strecke vor.

Am Colon descendens, das normalerweise locker und regelmäßig angeordnete Kotballen enthält, kommt es zu Verknikkungen und Verdrehungen, wenn dieses mit einem wurstförmigen Kotstrang prall gefüllt ist, wie dies bei Obstipationen beobachtet werden kann. Die dilatierten Darmschlingen nehmen am kleinen Kolon nahezu halbkreisförmige Konturen an. Die Verdrehung solcher aufeinanderfolgender Darmabschnitte um nur $180^{\circ}$ gegeneinander bewirkt bereits einen vollständigen Verschluss des Darmlumens.

Besonders markant ist die Knickung des Darmrohrs im Übergangsbereich zwischen dem Colon descendens mit seinem langen Gekröse und dem Rektum, welches nur ein kurzes Gekröse hat: z.B. beim sogenannten Ödem des terminalen Kleinkolons (Huskamp et al. 1982) und beim Ausfall der Peristaltik in diesem Bereich infolge embolisch-thrombotisch bedingter Mangeldurchblutung (Kopf 1983). Der Endteil des Colon descendens wird durch den sich aufstauenden Kot in die Excavatio rectogenitalis der Beckenhöhle gepresst, wodurch die nahezu 180gradige Knickung zum Rektum fixiert wird. Diese Retroflexio coli descendentis entsteht zwangsläufig auch bei Gekröseabrissen des Mesocolon descendens bei der Geburtsstute (Huskamp 1976, Kopf et al. 1979, Lorin und Kopf 1980), wo es durch den Ausfall der Peristaltik infolge Mangeldurchblutung zur Obstipation des abgerissenen terminalen Kleinkolons kommt.

Die Kombination zwischen Darmlähmung und sekundären Lageveränderungen infolge passiver Darmbewegungen wird als gemischter lleus bezeichnet. Im fortgeschrittenen Stadium eines Dünndarmileus kommt es am prästenotischen Darmschenkel fast immer zu diesem Zustand.

\section{Schlussfolgerung}

Die Analyse der Operations- und Sektionsbefunde sowie ergänzende Experimente und laparoskopische Beobachtungen haben es ermöglicht, dass wir uns heute ein ziemlich genaues Bild von den pathologischen Bewegungsvorgängen des Pferdedarmes machen können, die zur Entsehung einer Darmverlagerung oder -einklemmung führen. Dieser Einblick in die Pathogenese gibt uns wichtige Hinweise bezüglich der - vordem eher empirischen - Anweisungen an das Pflegepersonal und für unser therapeutisches Vorgehen. Um zu verhindern, dass sich eine mutmaßlich strangulierte Darmpartie rasch vergrößert, müssen schädliche aktive und passive Darmbewegungen gemildert oder vermieden werden. Um zu bewirken, dass die Abschnürung der Darmgefäße i.B. der Strangulationsortes abgesenkt wird, sollte der intraluminale Druck gesenkt werden. Daraus ergeben sich eine Reihe von Geboten und Maßnahmen:

- Initialer und wiederholter Einsatz von Spasmo-Analgetika ist in jedem Fall günstig.

- Bei Blähungen soll das Pferd am Niederlegen und Wälzen gehindert werden, da dadurch die Entstehung von Lageveränderungen begünstigt wird. (Ausnahme: Gesteuertes Wälzen bei nachweislicher Aufhängung der Grimmdarmschleife über dem Milznierenband).

- Drucksenkung im geblähten Magen (Nasenschlundsonde) und im Zäkum und allenfalls im großen Kolon (Zäkumpunktion) sind geeignete Mittel um die Strangulationsspannung herabzusetzen und somit die örtlichen Zirkulationsverhältnisse zu verbessern.

- Durch die -nötigenfalls wiederholte- Absenkung des intraabdominalen Druckes wird die Atmung (Reduktion der Zwerchfellhochstandes) sowie der venöse Rückfluss zum Herzen (V.cava caudalis) gefördert.

- Von der frühzeitigen bzw. unkritischen Eingabe von koterweichenden Mitteln (besonders salinischen Laxantien) bei unsicherer Diagnose wird nachdrücklich abgeraten: Vermehrte prästenotische Füllung beschleunigt die Mechanismen des Einsaugens, wodurch die von der Strangulation betroffene Darmpartie rascher vergrößert wird. Beim Durchwandern einer Einklemmung wird der anatomische Endpunkt rascher erreicht und die Abschnürung verstärkt.

Die Konkretisierung der Vorstellungen über die Vorgänge im Abdomen, die zu Darmverlagerungen führen, erleichtert ganz wesentlich die Interpretation der erhobenen Befunde und ermöglicht therapeutische und prognostische Konsequenzen.

\section{Literatur}

Dobberstein J. und Hartmann (1932) Über die Anastomosenbildung im Bereich der Blind- und Grimmdarmarterien des Pferdes und ihre Bedeutung für die Entstehung de embolischen Kolik. Berl. Tierärztl. Wschr. 1932, 397-402

Fischer A. T. jr.(1997) The Use of Laparoscopy in Horses with Colic. Proc.5.Kongress für Pferdemedizin und-chirurgie, 55,56. Genf, 14.-16.Dezember 1997

Grazl E. (1934) Die Pathogenese der Blinddarmverstopfungen des Pferdes. Wien.tierärztl.Mschr. 21, 481

Gratzl E. (1937) Beiträge zum Kolikproblem, V. Mitteilung: Welche Ursachen lösen die Darmkrämpfe bei den Koliken des Pferdes aus? Wien. Tierärztl. Mschr. 24, 33-43

Gratzl E. (1937) Beiträge zum Kolikproblem, VI. Mitteilung: Welche Ursachen lösen den Erregungszustand des Parasympathicus bei den Koliken aus? Wien. Tierärztl. Mschr. 24, 131-138

Gratzl E. (1942) Entstehung und Behandlung der Koliken des Pferdes. Dtsch. Tierärztl. Wschr. 50, 141-144

Huskamp B., Boening K. J., Becker M. und v. Plocki K. A. (1980) Die Ergebnisse operativer Kolikbehandlung, dargestellt am Patientengut des Jahres 1979 der Tierklinik Hochmoor. Proc. 7. Arbeitstagung, Fachgruppe Pferde-Krankheiten der Deutschen Veterinärmedizinischen Gesellschaft, Hamburg (1980)

Huskamp B., Daniels H. und Kopf N. (1983) Magen-Darm-Krankheiten. In: Dietz, O., und Wiesner, E. (Hrsg.): Handbuch der Pferdekrankheiten, VEB Gustav Fischer Verlag Jena, 2. Bd.

Huskamp B. (1976/1977) Mündliche Mitteilungen, unveröffentlicht Huskamp B. und Kopf N. (1980) Die Verlagerung des Colon ascen- 
dens in den Milznierenraum beim Pferd (Hernia spatii lienorenalis coli asdendentis ). Tierärztl. Prax. 8, 327-339, 495-506

Huskamp B. und Kopf N. (1983) Right Dorsal Displacement of the Large Colon in the Horse. Equine Pract. 5, 20-29

Huskamp B. und Kopf N. (1997) Hernia inguinalis incarcerata beim Pferd. Prakt. Tierarzt 78, 217

Jaksch W. (1982) Krankheiten des Verdauungsapparates. In: Wintzer, H.-J. (Hrsg.): Krankheiten des Pferdes, Verl. Paul Parey, BerlinHamburg.

Joest E. (1971) Handbuch der speziellen pathologischen Anatomie der Haustiere. Verl. Paul Parey, Berlin-Hamburg, 3. Aufl., Bd. VII.

Kopf N. (1976) Beitrag zur rektalen und intraperitonealen Diagnostik des chirurgisch behandelten Kolikpferdes. Diss. Med. Vet. Wien 1976

Kopf N. (1982) Zäkokolostomie bei einem Pferd. Wien. tierärztl. Mschr. 70, 107-108

Kopf N. (1984) Indikation, Ergebnisse und Aussichten operativer Kolikbehandlung beim Pferd - Eine Analyse von 149 klinischen Fällen. Wien. Tierärztl. Mschr. 71, 43-51, 121-133, 162-169

Kopf N. (1985) Über die Entstehung der Darmverlagerungen des Pferdes. Pferdeheilkunde 1, 131-148

Kopf N. (1987) Parasitär bedingte Koliken des Pferdes - Vorkommen, klinische Manifestation sowie Operations- und Sektionsbefunde. Pferdeheilkunde 3, 69-82

Kopf N., Niebaver G. W. und Rettenbacher G. (1979) Innere Verletzungen als Ursache oder Folge von lleus beim Pferd. Wien. Tierärztl. Mschr. 66, 233-247

Kopf N., Kreisel A., Rumpf W. und Vogel B. (1984) Dünndarmstrangulation infolge Gekrösemissbildung bei einem zweijährigen Araberhengst. Wien. Tierärzłl. Mschr. 71, 29-30

Kopf N., Pfeil L. und Mitterer T. (1984) Dünndarmeinklemmung im Foramen epiploicum und in einem vernarbten Riss im Gekröse des Colon descendens bei einem neuniährigen Vollblutwallach. Wien. Tierärztl. Mschr. 71, 382-383

Kopf N. und Zechner G. (1988) Funktionell-anatomische Studien zur Entstehung der sogenannten sanduhrförmigen Einziehung am Processus vaginalis des Hengstes bei der Hernia inguinalis incarcerata. Tagungsbericht der 10.Arbeitstagung der Fachgruppe "Pferdekrankheiten"der Deutschen Veterinärmedizinischen Gesellschaft in Wiesbaden, 177-188
Kopf N. (1997) in Robinson N. E.(Hrsg.) Current Therapy in Equine Medicine, Ed. 4, 170-174

Kopf N. (1997) Pathogenese der Darmverlagerung und der Darmeinklemmung beim Pferd. Proc. 5th Geneva Congress of Equine Medicine and Surgery. Genf, 14.-16.Dezember 1997; 115-118

Lorin D. und Kopf N. (1980) Abriss des Mesocolon descendens und partielle Mastdarmnekrose nach Mastdarmvorfall infolge Schwergeburt bei einer Kaltblutstute. Wien. Tierärztl. Mschr. 67, 47-50

Marek J. (1907) Folgen des Verschlusses der Gekrösearterien, Thrombose der Gekrösearterien. Arch. Tierheilkunde. 33, $225-$ 280

Möllmann L. H. (1932) Zur Pathologie der sympathischen Bauchganglien des Pferdes - Ganglion coeliacum plus mesentericum anterius - zugleich ein Beitrag zur Pathogenese der Kolik. Z. Veterinärkunde. 44, 273-288

Olt A. (1932) Das Aneurysma verminosum des Pferdes und seine unbekannten Beziehungen zur Kolik. Dtsch. Tierärzłl. Wschr., 326332

Pilwat F. (1910) Die pathologische Anatomie der Kolik des Pferdes. Arch. Wiss. Prakt. Tierheilk., Supplement, 436-572

Slocombe J. O. D., McCraw B. M., Pennock P. W. und Vasey J. (1982): Effectiveness of Ivermectin Against Later 4thStage Strongylus Vulgaris in Ponies. Am. J. Vet. Res. 43, 1525-1529

Überreiter O. (1958) Leistenbruch beim Hengst und beim Wallach, in Wirth, D.; Lexikon der Therapie und Prophylaxe für Tierärzte. Verlag Urban \& Schwarzenberg, Wien, Bd. II, 2. Aufl., 761-765

Wall S. (1908) Die Kolik des Pferdes. Evar Haeggströms Boktryckeri, A. B. - Stockholm

Zechner G. (1986) Beitrag zur Entstehung der sog. sanduhrförmigen Einziehung des Proc.vaginalis bei der Hernia inguinalis incarcerata des Hengstes. Diss. Med. Vet. Wien

Univ.Prof. Doz. Dr. Norbert Kopf

Breitenseer Strasse 16

1140 Wien

Österreich

dr.norbert.kopf@kleintierklinik-breitensee.at 